\title{
Tecnura
}

\section{Viabilidad de uso del polvo de vidrio como fundente en la elaboración de baldosas de gres porcelánico}

\section{Feasibility of using glass powder as flux in porcelain stoneware tille manufacturing}

\author{
Silvio Delvasto Arjona*, Álvaro Guzmán Aponte**, John Alexander Torres León***, \\ Martha Lorena Cedeño Venté****, David Felipe Acosta Guarín*****
}

Fecha de recepción: 29 de noviembre de 2013

Fecha de aceptación: 5 de diciembre de 2014

Citation / Para citar este artículo: Delvasto Arjona, S., Guzmán Aponte, Á., Torres León, J. A., Cedeño Venté, M. L., \& Acosta Guarín, D. F. (2015). Viabilidad de uso del polvo de vidrio como fundente en la elaboración de baldosas de gres porcelánico. Revista Tecnura, 19(44), 59-70. doi:http://dx.doi.org/10.14483/udistrital.jour. tecnura.2015.2.a04

\section{Resumen}

En este artículo se presentan los resultados de una investigación sobre el uso del polvo de vidrio como remplazo del feldespato en la fabricación de gres porcelánico. Se prepararon mezclas en donde el polvo de vidrio sustituyó al feldespato en dos volúmenes distintos (25\% y $50 \%)$. Especímenes de las pastas obtenidas fueron moldeados, secados y cocidos. Las propiedades físicas (densidad aparente, porosidad, absorción, entre otras) y mecánicas (resistencia a flexión) de los especímenes cocidos estándar (V0) se compararon con las de especímenes cocidos en donde el material fundente fue sustituido en la formulación por polvo de vidrio en porcentajes de $25 \%$ y $50 \%$ (V25 y V50). Se encontró que la adición de polvo de vidrio en las formulaciones ocasionaba disminución en la resistencia a la flexión e incremento en los valores de absorción de agua. Sin embargo, la acción fundente del polvo de vidrio disminuyó la temperatura de sinterización respecto a la mezcla estándar en $150{ }^{\circ} \mathrm{C}$ para remplazos de $50 \%$ y $25 \%$, respectivamente. Mediante MEB se evidenció en la microestructura de las piezas cocidas (V0, V25 y V50) la presencia de cristales de mullita primaria, adyacentes a cristales de mullita secundaria (agujas elongadas).

Palabras clave: feldespato, gres porcelánico, polvo de vidrio, vidrio.

\begin{abstract}
This article presents the results of a research on the use of glass powder as substitute of feldspar for manufacturing stoneware tiles. Mixtures were prepared in which glass powder substituted feldspar in two
\end{abstract}

\footnotetext{
* Ingeniero químico, Master of Science en Materiales de Ingeniería Civil y Manejo de Materiales, doctor en Ingeniería de Nuevos Materiales y sus Tecnologías de Fabricación. Docente de la Universidad del Valle. Cali, Colombia. Contacto: silvio.delvasto@correounivalle.edu.co

** Ingeniero de Materiales, doctor en Ingeniería, Área de Énfasis en Ingeniería de Materiales, docente de la Escuela Colombiana de Ingeniería Julio Gravito, Bogotá, Colombia. Contacto: alvaro.guzman@escuelaing.edu.co

*** Ingeniero de Materiales, estudiante de maestría en Ingeniería Física en curso, Universidad del Cauca, Popayán, Colombia. Contacto:johntl@unicauca.edu.co

**** Ingeniera de Materiales, estudiante de maestría en Metalurgia y Ciencia de los Materiales, Universidad Pedagógica y Tecnológica de Colombia,Tunja, Colombia. Contacto: martha.cedeno@uptc.edu.co

***** Estudiante de Ingeniería de Materiales, Universidad del Valle, Cali, Colombia. guacos12@gmail.com
} 
different percentages (25 wt \% and $50 \mathrm{wt} \%$ ). Specimens of the obtained mixtures were molded, dried and sintered. Physical (apparent density, porousness, absorption, among others) and mechanical properties (flexion resistance) of standard sintered bodies (V0) were compared to the sintered bodies in which the melting material was substituted in the formulation by glass powder in percentages of $25 \%$ and $50 \%$ (V25 and V50). It was found that the glass powder addition in the formulations caused decrease in flexion resistance and increase in water absorption values. However, the melting action of glass powder reduced the sintering temperature in regards with the standard mixture in $150{ }^{\circ} \mathrm{C}$ for substitutions of $50 \%$ and $25 \%$, respectively. By using Scanning Electron Microscopy (SEM) needles of primary mullite and secondary mullite (elongated needles) in a vitreous phase were identified.

Keywords: feldspar, glass, glass powder, porcelain stoneware tile.

\section{INTRODUCCIÓN}

El gres porcelánico es un producto cerámico caracterizado por una baja absorción de agua $\leq$ 0,5\% y una resistencia mecánica a flexión $>35$ MPa acorde con la norma ISO 13006. La baldosa de gres porcelánico es una baldosa cerámica totalmente vitrificada, impermeable, esmaltada o no, cuyo soporte en cocido es blanco o coloreado artificialmente; compuesta de una mezcla triaxial que contiene caolín (o arcillas caoliníticas), cuarzo y feldespato (Cuéllar Lozano, 2000); (Sánchez, 2002).

Los recubrimientos cerámicos han experimentado un notable crecimiento en los últimos años, debido a sus excelentes propiedades mecánicas y decorativas. A nivel mundial, la mayor demanda se dirige hacia lo que se denomina baldosa de gres porcelánico, la cual ha experimentado un crecimiento en la producción y las ventas, en comparación con otros materiales cerámicos de construcción; esto se atribuye a sus altas propiedades tecnológicas, especialmente lo que se refiere a la absorción de agua, resistencia química y resistencia al hielo y a las propiedades mecánicas como la resistencia a la flexión y a la abrasión (Sánchez et al., 2006); (Márquez, Rincón, \& Romero, 2008); (De Noni Jr., Hotza, Cantavella, \& Sánchez, 2008); (Márquez, De la Torre, Aranda, Rincón, \& Romero, 2009); (Ríos R, 2009);
(Márquez, Rincón, \& Romero, 2010a); (Zanelli, Raimondo, Guarini, \& Dondi, 2011). Pese a esto, el costo de este material puede superar considerablemente al de la cerámica tradicional, ya que su obtención requiere riguroso control microestructural, materias primas de alta calidad y un proceso de fabricación altamente tecnificado (Sánchez, 2003). Por esta razón, en la actualidad se trabaja, por ejemplo, en la implementación de materias primas alternativas a las convencionales que permitan disminuir los costos de producción y mantener o mejorar las propiedades tanto físicas como mecánicas. Teniendo en mente este interés, el proceso de reutilización y reciclaje de materiales sería interesante de estudiar ya que podría permitir obtener productos a menores costos, además de ayudar a proteger el medio ambiente.

La industria cerámica ha utilizado con este objetivo distintos materiales como cenizas de carbón, de cascarilla de arroz, residuos de vidrios, entre otros, que se consideran "desechos". En mezclas de cerámica blanca, por ejemplo, en donde el feldespato actúa como fundente, Guzmán, Delvasto, Sánchez \& Amigó (2013) encontraron que la ceniza de tamo de arroz (CTA) puede remplazar parcialmente al feldespato utilizado para la elaboración de pastas de loza.

Otros investigadores como Matteucci, Dondi, \& Guarini (2002); Braganca \& Bergmann (2004); 
Tucci, Esposito, Rastelli, Palmonari, \& Rambaldi (2004); Luz \& Ribeiro (2007); Rambaldi, Carty, Tucci, \& Esposito (2007); Raimondo, y otros (2007); Andreola, Barbieri, Karamanova, LanceIlotti, \& Pelino (2008) han hecho uso de diferentes desechos de vidrio (tubos de rayos catódicos (CRT (Panel Cathode Ray Tube)), sílice-soda-cal (soda-lime-silica, SLS, glass), entre otros), como remplazo del material fundente en la producción de lozas, cerámica tradicional y gres porcelánico, y encontraron algunos resultados interesantes (acelera el proceso de densificación, disminución en la temperatura óptima de cocción, entre otros). Sin embargo, otros autores como Andreola, Barbieri, Corradi, \& Lancellotti (2007) mencionan que el uso de vidrio CRT en la industria cerámica aún no es conveniente, debido a carencias y limitaciones en el reciclaje de sus residuos, ya que estos residuos poseen elementos peligrosos como $\mathrm{Pb}, \mathrm{Cd}, \mathrm{P}$, etc., con efecto negativo para el reciclador y el ambiente.

En esta investigación, el material fundente (feldespato sódico-potásico) de una composición de gres porcelánico fue sustituido por polvo de vidrio de ventana (sódico-cálcico). La razón de esta selección fue sustentada en el hecho de que el polvo de vidrio se encuentra compuesto por óxidos alcalinos $\mathrm{K}_{2} \mathrm{O}$ y $\mathrm{Na}_{2} \mathrm{O}$, además de óxidos alcalinotérreos como $\mathrm{CaO}$ y $\mathrm{MgO}$, elementos eficientes para promover la fundición y formación de fase líquida. A partir de ello, el propósito de este trabajo fue estudiar la posibilidad de usar el polvo de vidrio como remplazo del material fundente tradicional empleado en la producción de gres porcelánico, a través de experimentos de laboratorio y basados en el comportamiento a cocción del producto en verde y las propiedades físico-mecánicas del material sinterizado. En resumen, en este trabajo se estudió el uso de un "residuo" que podría generar beneficio económico y reducción del impacto sobre el medio ambiente.

\section{MATERIALES Y MÉTODOS}

\section{Materiales}

El vidrio empleado en esta investigación es vidrio de ventana. Las demás materias primas utilizadas para la elaboración de las pastas cerámicas triaxiales fueron cuarzo, feldespato, arcilla y caolín, que se comercializan en Colombia por la empresa Quimex Industriales S.A. El vidrio fue acondicionado a través de un proceso de lavado y molturación en molino de discos a 10 pasadas y en molino de bolas por un tiempo de 1 hora. Los diámetros medios de partícula del vidrio molido, arcilla, caolín, feldespato y cuarzo fueron $35,69 \mu \mathrm{m}, 12,86 \mu \mathrm{m}$, $27,88 \mu \mathrm{m}, 20,68 \mu \mathrm{m}$ y $20,43 \mu \mathrm{m}$, respectivamente, valores que se determinaron empleando un equipo de Granulometría Láser Mastersizer 2000, versión 5.22. El tamaño de partícula del cuarzo se encontró en el rango de [15 - $30 \mu \mathrm{m}]$, rango recomendado por la literatura para la máxima resistencia de la porcelana (Mattyasovszky-Zsolnay, 1957); (Carty \& Senapati, 1998); (Stathis, Ekonomakou, Stournaras, \& Ftikos, 2004); (Braganca, Bergmann, \& Hubner, 2006). Las composiciones química y mineralógica de las materias primas se identificaron mediante las técnicas de fluorescencia de rayos $X(F R X)$ y difracción de rayos $X$ (DRX) (ver la tabla 1 y la tabla 2), empleando un espectrómetro de fluorescencia de rayos X, MagixPro PW - 2440 Philips y un difractómetro BRUKER Theta-Theta modelo D8 Advance, respectivamente.

\section{Procesamiento del gres porcelánico estándar y adicionado con polvo de vidrio}

Se empleó una formulación estándar (V0) constituida por $25 \%$ de arcilla, $15 \%$ de caolín, 15\% de cuarzo y $45 \%$ de feldespato. Con base en esta se prepararon dos formulaciones adicionales, en las cuales la proporción de feldespato fue remplazada en $25 \%$ y $50 \%$ por polvo de vidrio (V25 y V50). La 
Tabla 1. Composición química de las materias primas.

\begin{tabular}{cccccc}
\hline \multirow{2}{*}{$\begin{array}{c}\text { Compuesto y/o } \\
\text { elemento }\end{array}$} & Caolín & Feldespato & Cuarzo & Arcilla & Polvo de vidrio \\
\cline { 2 - 6 } & 70,22 & 77,33 & 96,88 & 46,36 & 77,24 \\
\hline $\mathrm{SiO}_{2}$ & 19,65 & 12,52 & 1,93 & 36,94 & 1,65 \\
\hline $\mathrm{Al}_{2} \mathrm{O}_{3}$ & 1,22 & 0,23 & 0,18 & 1,05 & 0,00 \\
\hline $\mathrm{Fe}_{2} \mathrm{O}_{3}$ & 0,00 & 0,42 & 0,00 & 0,15 & 5,34 \\
\hline $\mathrm{CaO}$ & 0,38 & 0,00 & 0,10 & 0,20 & 0,08 \\
\hline $\mathrm{MgO}$ & 0,09 & 4,20 & 0,00 & 0,00 & 15,36 \\
\hline $\mathrm{Na}_{2} \mathrm{O}$ & 2,34 & 4,94 & 0,00 & 0,39 & 0,33 \\
\hline $\mathrm{K}_{2} \mathrm{O}$ & 0,08 & 0,00 & 0,06 & 0,61 & 0,00 \\
\hline $\mathrm{TiO}_{2}$ & 0,05 & 0,00 & 0,00 & 0,00 & 0,00 \\
\hline $\mathrm{MnO}_{\mathrm{P} O} \mathrm{O}_{5}$ & 0,00 & 0,00 & 0,00 & 0,03 & 0,00 \\
\hline $\mathrm{Cl}$ & 0,00 & 0,00 & 0,00 & 0,00 & 0,00 \\
\hline $\mathrm{S}$ & 0,00 & 0,00 & 0,00 & 0,00 & 0,00 \\
\hline $\mathrm{Zn}$ & 0,00 & 0,00 & 0,00 & 0,01 & 0,00 \\
\hline $\mathrm{Rb}$ & 0,01 & 0,02 & 0,00 & 0,00 & 0,00 \\
\hline $\mathrm{Br}$ & 0,00 & 0,00 & 0,00 & 0,00 & 0,00 \\
\hline $\mathrm{Cu}$ & 0,00 & 0,00 & 0,00 & 0,00 & 0,00 \\
\hline $\mathrm{Ba}$ & 0,10 & 0,00 & 0,00 & 0,00 & 0,00 \\
\hline $\mathrm{Zr}$ & 0,00 & 0,00 & 0,00 & 0,01 & 0,00 \\
\hline $\mathrm{PF}$ & 5,86 & 0,35 & 0,85 & 14,25 & - \\
\hline
\end{tabular}

Fuente: elaboración propia.

Tabla 2. Composición mineralógica de las materias primas.

\begin{tabular}{|c|c|c|c|c|}
\hline \multirow{5}{*}{$\begin{array}{c}\text { Fases } \\
\text { mineralógicas } \\
\text { (ICDD) }\end{array}$} & Caolín & Feldespato & Cuarzo & Arcilla \\
\hline & Cuarzo $\left(\mathrm{SiO}_{2}\right)$ & Albita $\left(\mathrm{NaAlSi}_{3} \mathrm{O}_{8}\right)$ & Fase mayoritaria: Cuarzo $\left(\mathrm{SiO}_{2}\right)$ & Cuarzo $\left(\mathrm{SiO}_{2}\right)$ \\
\hline & $\begin{array}{l}\text { Caolinita (Al- } \\
\left.{ }_{2} \mathrm{Si}_{2} \mathrm{O}_{5}(\mathrm{OH})_{4}\right)\end{array}$ & $\begin{array}{l}\text { Feldespato potásico } \\
\left(\mathrm{KAISi}_{3} \mathrm{O}_{8}\right)\end{array}$ & $\begin{array}{l}\text { Fase minoritaria: Caolinita } \\
\left(\mathrm{Al}_{2} \mathrm{Si}_{2} \mathrm{O}_{5}(\mathrm{OH})_{4}\right)\end{array}$ & Caolinita $\left(\mathrm{Al}_{2} \mathrm{Si}_{2} \mathrm{O}_{5}(\mathrm{OH})_{4}\right)$ \\
\hline & $\begin{array}{l}\text { Illita/M. Moscovita } \\
\left(\mathrm{KAI}_{2} \mathrm{Si}_{3} \mathrm{AlO}_{10}(\mathrm{OH})_{2}\right)\end{array}$ & Cuarzo $\left(\mathrm{SiO}_{2}\right)$ & Trazas: Nefelina $\left((\mathrm{Na}, \mathrm{K}) \mathrm{AlSiO}_{4}\right)$ & $\begin{array}{l}\text { Illita/M. Moscovita (KAI- } \\
\left.{ }_{2} \mathrm{Si}_{3} \mathrm{AlO}_{10}(\mathrm{OH})_{2}\right)\end{array}$ \\
\hline & & $\begin{array}{l}\text { Illita/M. Moscovita } \\
\left(\mathrm{KAI}_{2} \mathrm{Si}_{3} \mathrm{AlO}_{10}(\mathrm{OH})_{2}\right)\end{array}$ & & Gibbsita $\left(\mathrm{Al}(\mathrm{OH})_{3}\right)$ \\
\hline
\end{tabular}

Fuente: elaboración propia.

composición química de las mezclas se encuentra reportada en la tabla 3.

Se preparó $1 \mathrm{~kg}$ de cada muestra por molienda en seco mediante el uso de un molino de bolas, utilizando como medio de molienda cuerpos moledores de alúmina con geometría cilíndrica, durante un tiempo de 30 minutos. 25 g de cada mezcla semiseca ( $6 \%$ agua) se prensaron uniaxialmente en un molde de acero a $50 \mathrm{MPa}$ en especímenes prismáticos de 117,0 mm de largo $\times 27,0 \mathrm{~mm}$ de ancho y $4 \mathrm{~mm}$ de espesor. Los especímenes se secaron en un horno mufla a 110 ${ }^{\circ} \mathrm{C}$ y posteriormente fueron cocidos en un horno eléctrico de laboratorio (Carbolite RHF 1600) a una rampa de calentamiento de $20{ }^{\circ} \mathrm{C} / \mathrm{min}$ entre $25^{\circ} \mathrm{C}$ y $600{ }^{\circ} \mathrm{C}$ y un tiempo de sostenimiento de 6 minutos; y $20{ }^{\circ} \mathrm{C} / \mathrm{min}$ desde $600{ }^{\circ} \mathrm{C}$ a la temperatura máxima de cocción y un tiempo 
Tabla 3. Composición química de las mezclas (\% en peso total).

\begin{tabular}{cccc}
\hline \multirow{2}{*}{ Compuesto y/o elemento } & \multicolumn{3}{c}{ Mezcla (\% peso total) } \\
\cline { 2 - 4 } & $\mathbf{V 0}$ & $\mathbf{V 2 5}$ & V50 \\
\hline $\mathrm{SiO}_{2}$ & 71,45 & 71,44 & 71,43 \\
\hline $\mathrm{Al}_{2} \mathrm{O}_{3}$ & 18,11 & 16,88 & 15,66 \\
\hline $\mathrm{Fe}_{2} \mathrm{O}_{3}$ & 0,58 & 0,55 & 0,52 \\
\hline $\mathrm{CaO}$ & 0,23 & 0,78 & 1,33 \\
\hline $\mathrm{MgO}$ & 0,12 & 0,13 & 0,14 \\
\hline $\mathrm{Na}_{2} \mathrm{O}$ & 1,90 & 3,16 & 4,41 \\
\hline $\mathrm{K}_{2} \mathrm{O}$ & 2,67 & 1,63 \\
\hline $\mathrm{TiO}_{2}$ & 0,17 & 0,17 \\
\hline $\mathrm{MnO}_{2}$ & 0,01 & 0,01 \\
\hline $\mathrm{P}_{5}$ & 0,01 & 0,01 \\
\hline $\mathrm{Cl}$ & 0,00 & 0,01 & 0,00 \\
\hline $\mathrm{S}$ & 0,00 & 0,01 & 0,00 \\
\hline $\mathrm{Zn}$ & 0,00 & 0,00 \\
\hline $\mathrm{Rb}$ & 0,01 & 0,00 \\
\hline $\mathrm{Sr}$ & 0,01 & 0,01 \\
\hline $\mathrm{Cu}$ & 0,00 & 0,00 \\
\hline $\mathrm{Ba}$ & 0,00 & 0,01 & 0,00 \\
\hline $\mathrm{Zr}$ & 0,02 & 0,02 \\
\hline $\mathrm{PF}$ & 0,00 & 0,00 & 0,00 \\
\hline
\end{tabular}

Fuente: elaboración propia.

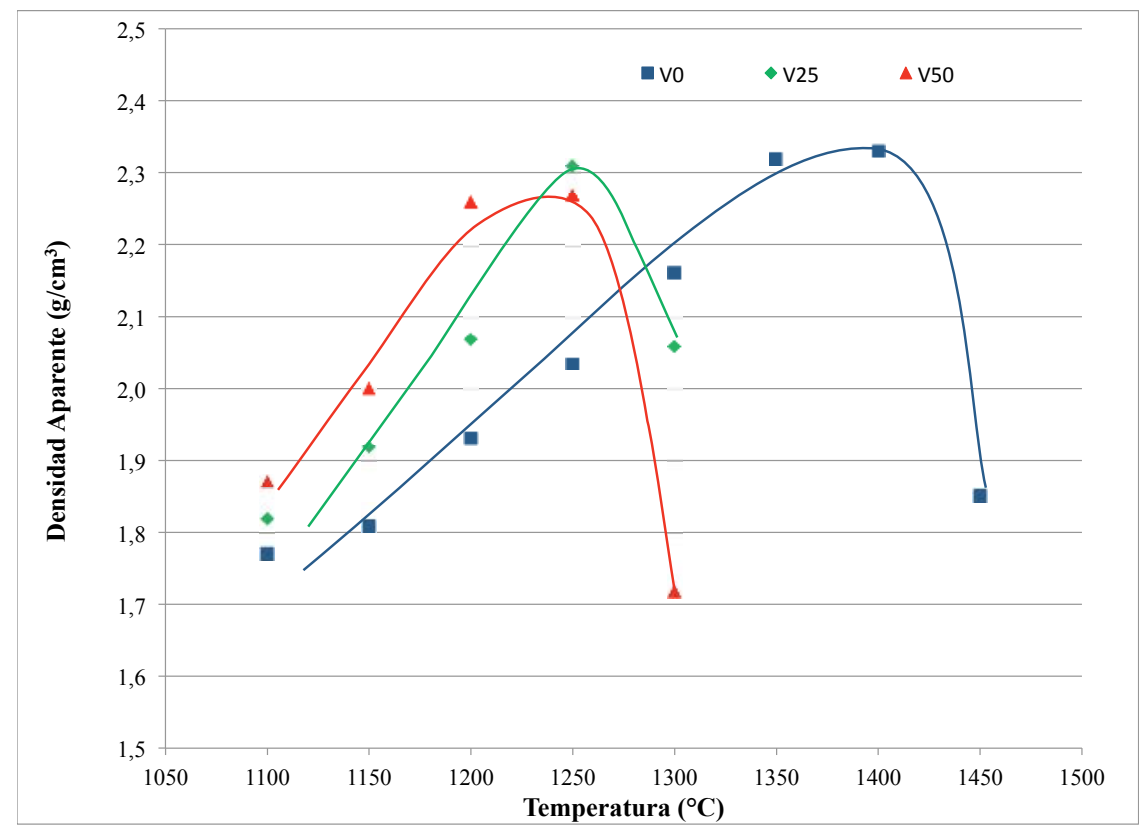

Figura 1. Densidad aparente de las probetas cocidas $\vee 0(\boldsymbol{\bullet}), \mathrm{V} 25(\diamond)$ y $\mathrm{V} 50(\mathbf{\Delta})$, en función de la temperatura de cocción.

Fuente: elaboración propia. 
de sostenimiento de 6 minutos. El enfriamiento de los especímenes cocidos se realizó dentro del horno, con el fin de evitar tensiones residuales macroscópicas (De Noni Jr., Hotza, Cantavella, \& Sánchez, 2010). Las temperaturas máximas de cocción se encontraron en el rango de 1100 ${ }^{\circ} \mathrm{C}-1450{ }^{\circ} \mathrm{C}$ (dependiendo de la composición), a intervalos de $50{ }^{\circ} \mathrm{C}$. La temperatura de máxima densificación fue determinada, para cada mezcla, a partir del gráfico construido densidad aparente en cocido en función de la temperatura máxima de sinterización (ver la figura 1), haciendo uso de las condiciones experimentales descritas previamente.

Las mezclas de polvos cerámicos de gres porcelánico se sometieron a análisis termo-gravimétrico y térmico-diferencial (ATG, ATD) usando para ello un equipo TA Instruments, SDT-Q600; con una rampa de calentamiento de $10{ }^{\circ} \mathrm{C} / \mathrm{min}$, hasta una temperatura de $1100{ }^{\circ} \mathrm{C}$, bajo un flujo de gas (nitrógeno) de $100 \mathrm{ml} / \mathrm{min}$.

Se evaluó en las piezas cocidas el grado de vitrificación mediante ensayos de contracción lineal, porosidad, absorción y densidad conforme a los requerimientos de las normas ASTM C326, 2009; ASTM C329, 1988 (2006) y ASTM C373, 1988 (2006). Finalmente, se determinó la resistencia a la flexión considerando lo que señala la norma establecida para tal fin ASTM C674, 1988 (2006), empleando una máquina universal de ensayos INSTRON 3369, mediante un montaje a flexión en tres puntos con una distancia entre apoyos de 98 mm y una velocidad de aplicación de la carga de $1 \mathrm{~mm} / \mathrm{min}$. Para tener mayor confiabilidad de los datos obtenidos, se hicieron en promedio tres mediciones. Las características microestructurales de las piezas sinterizadas se observaron mediante microscopía electrónica de barrido (MEB), empleando un equipo Jeol JSM-6490LV; para ello la superficie de fractura de cada muestra fue pulida y atacada con una solución de ácido fluorhídrico (HF) a 5\% durante $3 \mathrm{~min}$, posteriormente fue lavada con agua destilada y alcohol etílico y finalmente secada y recubierta con $\mathrm{Au}-\mathrm{Pd}$.

\section{RESULTADOS Y DISCUSIONES}

\section{Caracterización de las materias primas}

Los resultados de la composición química de las materias primas empleadas (ver la tabla 1), permitieron evidenciar como constituyentes principales la sílice $\left(\mathrm{SiO}_{2}\right)$ y la alúmina $\left(\mathrm{Al}_{2} \mathrm{O}_{3}\right)$. En el caso de la arcilla y el caolín se observó además la presencia de óxido de hierro $\left(\mathrm{Fe}_{2} \mathrm{O}_{3}\right)$ en porcentajes de $1,05 \%$ y $1,22 \%$, respectivamente. El cuarzo presentó contenidos de $\mathrm{Fe}_{2} \mathrm{O}_{3}$ de $0,18 \%$ y de $\mathrm{TiO}_{2}$ de $0,06 \%$. Aunque la presencia de este tipo de óxidos genera coloración (cromóforos), algunos investigadores como Biffi, G. (2002) han empleado arcillas con porcentajes de $\mathrm{Fe}_{2} \mathrm{O}_{3}$ superiores a los de este estudio, obteniendo resultados favorables.

Con respecto al feldespato, se observó presencia de porcentajes similares en los contenidos de óxidos de $\mathrm{K}_{2} \mathrm{O}$ y $\mathrm{Na}_{2} \mathrm{O}$ (4,94\% y 4,20\%, respectivamente) y pequeñas concentraciones de $\mathrm{CaO}$ $(0,42 \%)$, motivo por el cual fue clasificado como sódico-potásico, corroborando los resultados obtenidos con DRX (ver la tabla 2). Por otra parte, las altas concentraciones de $\mathrm{Na}_{2} \mathrm{O}$ y $\mathrm{CaO}$ presentes en el polvo de vidrio confirman su naturaleza sódico-cálcica, ideal para vidrios de ventana.

\section{Evaluación de la aptitud como fundente del polvo de vidrio en la mezcla triaxial}

El remplazo del feldespato por polvo de vidrio en la mezcla ocasiona que los contenidos de $\mathrm{Al}_{2} \mathrm{O}_{3}$ y $\mathrm{K}_{2} \mathrm{O}$ disminuyan, mientras los contenidos de $\mathrm{Na}_{2} \mathrm{O}$ y $\mathrm{CaO}$ incrementan (ver la tabla 3 ).

Los ensayos de análisis térmico (ATG/ATD) de las mezclas de polvos de composición de gres porcelánico V0, V25\% y V50\%, mostraron un pico endotérmico en el rango de $0-200{ }^{\circ} \mathrm{C}$ atribuible a la remoción de agua adsorbida, seguido por la deshidroxilación del caolín $\left(494{ }^{\circ} \mathrm{C}\right.$ para V0, $496{ }^{\circ} \mathrm{C}$ para V25 y $495^{\circ} \mathrm{C}$ para V50) (ver la figura 2).

Se observó un pico endotérmico asociado a la transformación de cuarzo $\alpha$ a $\beta$ a $569{ }^{\circ} \mathrm{C}$ para V0, y 
$568{ }^{\circ} \mathrm{C}$ para V25 y V50. Finalmente, a $988^{\circ} \mathrm{C}$ para V0, y $985^{\circ} \mathrm{C}$ para V25 y V50, se evidenció un pico exotérmico asociado a la transformación de metacaolín a espinela o a fase premullita.

A partir de las curvas de máxima densificación de cada una de las mezclas V0, V25 y V50 (ver la figura 1), las temperaturas óptimas de cocción fueron $1400{ }^{\circ} \mathrm{C}, 1250{ }^{\circ} \mathrm{C}$ y $1250{ }^{\circ} \mathrm{C}$, respectivamente. Los valores óptimos de sinterización indicados anteriormente son coherentes con el rango de vitrificación óptima, el cual se logra cuando la porosidad abierta alcanza el valor mínimo y la contracción lineal es máxima, como se ilustra en las curvas de gresificación (ver la figura 3).

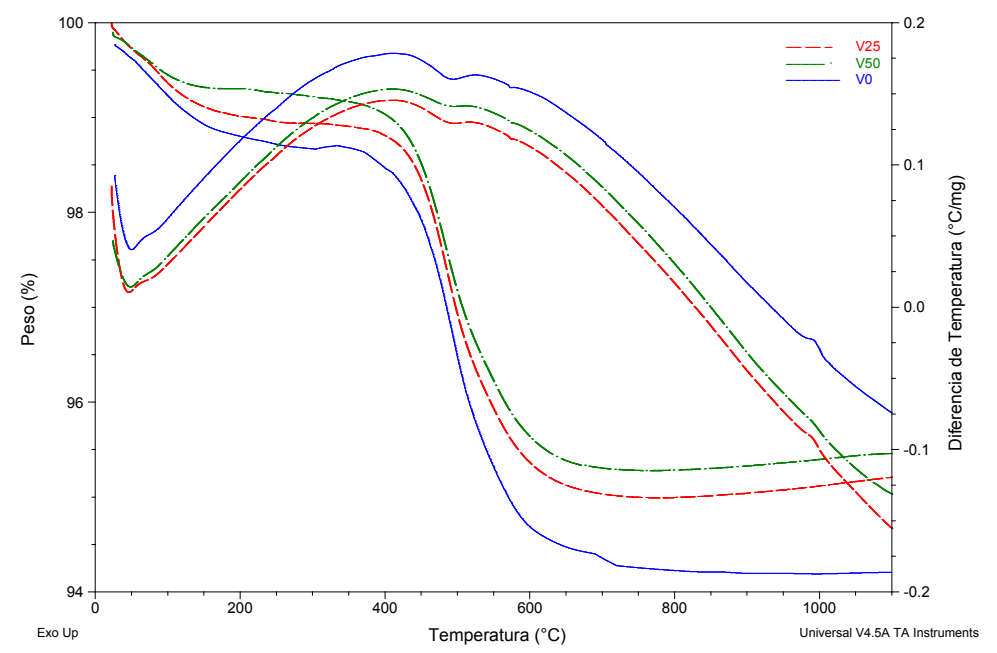

Figura 2. Curvas de ATG/ATD de mezclas de polvos de composición de gres porcelánico cuyas pastas fueron dosificadas con $0 \%$ de polvo de vidrio (V0), $25 \%$ de polvo de vidrio (V25) y $50 \%$ de polvo de vidrio (V50).

Fuente: elaboración propia.

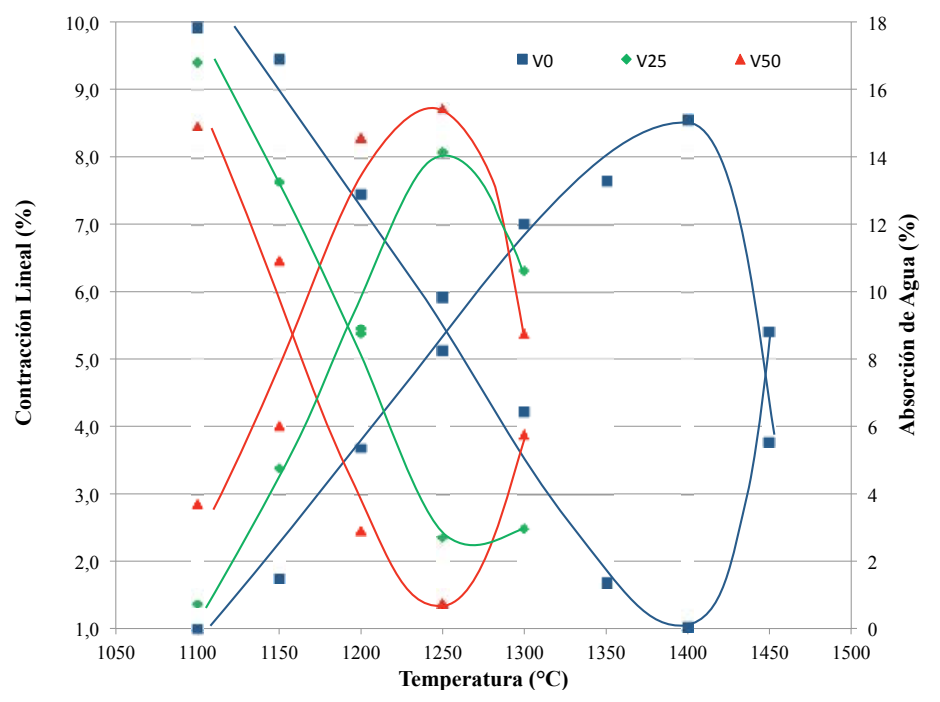

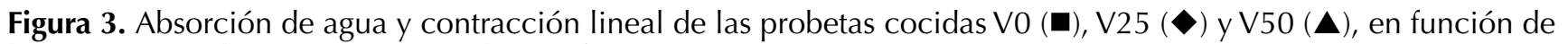
la temperatura de cocción (curvas de gresificación).

Fuente: elaboración propia. 
A partir de los resultados de las curvas de gresificación se observa que aquellas composiciones con sustitución de feldespato por polvo de vidrio (V25 y V50) presentan un rango de vitrificación óptima ligeramente más estrecho al mostrado por la composición estándar (V0), lo que indica que estas composiciones deben tener un control más riguroso durante su sinterización. La cocción por encima del rango de vitrificación óptima ocasiona un detrimento drástico de las propiedades físicas debido a la expulsión forzosa de los gases atrapados, generando ampollas (blisters) e hinchamiento (bloating) (Márquez, Rincón, \& Romero, 2008). Cabe mencionar que las temperaturas óptimas de cocción encontradas son superiores a las empleadas en la práctica industrial $\left(1180-1220{ }^{\circ} \mathrm{C}\right.$ ) (Raimondo y otros, 2009); (De Noni Jr., Hotza, Cantavella, \& Sánchez, 2010), lo cual podría atribuirse al uso de un feldespato sódico-potásico de escasa fundencia. Además, el equipamiento e instalaciones disponibles a nivel industrial generalmente no se encuentran a escala de laboratorio y, por tanto, se necesitan mayores condiciones de cocción (tiempo y temperatura) para alcanzar las propiedades tecnológicas comerciales. A pesar de lo anterior, es evidente que el incremento en los contenidos de $\mathrm{CaO}$ y $\mathrm{Na}_{2} \mathrm{O}$ en aquellas composiciones con sustitución de feldespato por polvo de vidrio (V25 y V50) favorece la vitrificación temprana de las composiciones cerámicas, permitiendo la producción de piezas cocidas con máxima densidad a una temperatura más baja que la de la composición estándar (V0) (ver la tabla 4).

Este comportamiento está en concordancia con lo mencionado por investigadores como Das \& Dana (2003); Zanelli, Raimondo, Dondi, Guarini, \& Tenorio (2004); Dana, Dey, \& Das (2005) y Yuruyen \& Toplan (2009), quienes indican que las composiciones cerámicas ricas en $\mathrm{Na}$ son menos viscosas que aquellas ricas en $\mathrm{K}$, facilitando la máxima densificación de las piezas cocidas. La óptima cocción a una temperatura inferior es una ventaja para las composiciones sustituidas con polvo de vidrio, confirmando el carácter fundente fuerte del mismo; sin embargo, los porcentajes de porosidad abierta y absorción de agua para las piezas cocidas V25 y V50 fueron mayores, comparadas con la mezcla estándar V0 (ver la tabla 4).

Braganca \& Bergmann (2004) mencionan que la densidad aparente en cocido es una propiedad que tiene una fuerte influencia sobre el módulo de ruptura de las piezas; por lo que, generalmente, a mayor densidad aparente se presenta mayor módulo de ruptura. En coherencia con lo anterior, los datos indicados en la figura 4 de resistencia a la flexión de las piezas sinterizadas en este trabajo ponen en evidencia una tendencia similar con los resultados obtenidos en la densidad aparente en cocido (ver la tabla 4).

Tabla 4. Propiedades físicas de las piezas en cocido obtenidas a la temperatura óptima de cocción

\begin{tabular}{lccc}
\hline \multicolumn{1}{c}{ Propiedades físicas } & V0 & V25 & V50 \\
\hline Temperatura óptima de cocción $\left({ }^{\circ} \mathrm{C}\right)$ & 1400 & 1250 & 1250 \\
\hline Contracción lineal $(\%)$ & 8,6 & 8,1 & 8,7 \\
\hline Densidad aparente en cocido $\left(\mathrm{g} / \mathrm{cm}^{3}\right)$ & 2,33 & 2,23 & 2,31 \\
\hline Porosidad abierta $(\%)$ & 0,1 & 6,3 & 1,8 \\
\hline Porosidad cerrada $(\%)$ & 4,6 & 0,6 & 5,9 \\
\hline Porosidad total $(\%)$ & 4,9 & 6,8 & 7,7 \\
\hline Absorción de agua $(\%)$ & 0,1 & 2,7 & 0,8 \\
\hline
\end{tabular}

Fuente: elaboración propia. 
Estos resultados, además, están de acuerdo con lo mencionado por Márquez, Rincón, \& Romero (2010a), quienes indican que la resistencia a la flexión no depende de la porosidad cerrada pero sí está relacionada con la porosidad abierta, ya que cuando la porosidad abierta disminuye se incrementa la resistencia a la flexión. Otros investigadores como Sánchez et al. (2006) también han observado un comportamiento similar.

Con base en los resultados de resistencia a flexión (ver la figura 4) y absorción de agua (ver la tabla 4) y basados en los criterios de la normativa ISO 13006 para la clasificación baldosas cerámicas prensadas en seco, los especímenes cocidos en este trabajo, en donde el feldespato fue sustituido por polvo de vidrio (V25 y V50), se deben considerar como semi-gres perteneciente al grupo Blla (resistencia a flexión > 22 MPa y absorción de agua $3-6 \%$ ) y gres perteneciente al grupo Blb (resistencia a flexión > 30 MPa y absorción de agua $0,5-3 \%)$, respectivamente. Mientras aquellos especímenes obtenidos a partir de los componentes tradicionales se consideran como gres porcelánico perteneciente al grupo Bla (resistencia a flexión > 35 MPa y absorción de agua $\leq 0,5 \%$ ).

Las micrografías de las superficies pulidas tomadas a 100X de MEB, en modo electrones secundarios (ver la figura 5 ( $a, b$ y c)), permitieron evidenciar una mayor porosidad cerrada en aqueIlas piezas cocidas V50 respecto a V0 y V25, corroborando los resultados obtenidos de porosidad cerrada indicados en la tabla 4.

Teniendo en cuenta lo mencionado por Tucci et al. (2004) es de esperar que aquellas piezas cocidas con polvo de vidrio, debido a la disminución del contenido de alúmina, se favorezca en menor proporción la formación de mullita. Sin embargo, las micrografías a 10000X de MEB en modo electrones secundarios (ver la figura 5 (d, e y f)), permitieron evidenciar en la microestructura de todas las piezas cocidas (V0, V25 y V50) la presencia de cristales de mullita primaria, adyacentes a cristales de mullita secundaria (agujas elongadas). Lo anterior, en concordancia con lo mencionado por Márquez, Rincón, \& Romero (2010b), quienes sugieren que los cristales de mullita primaria

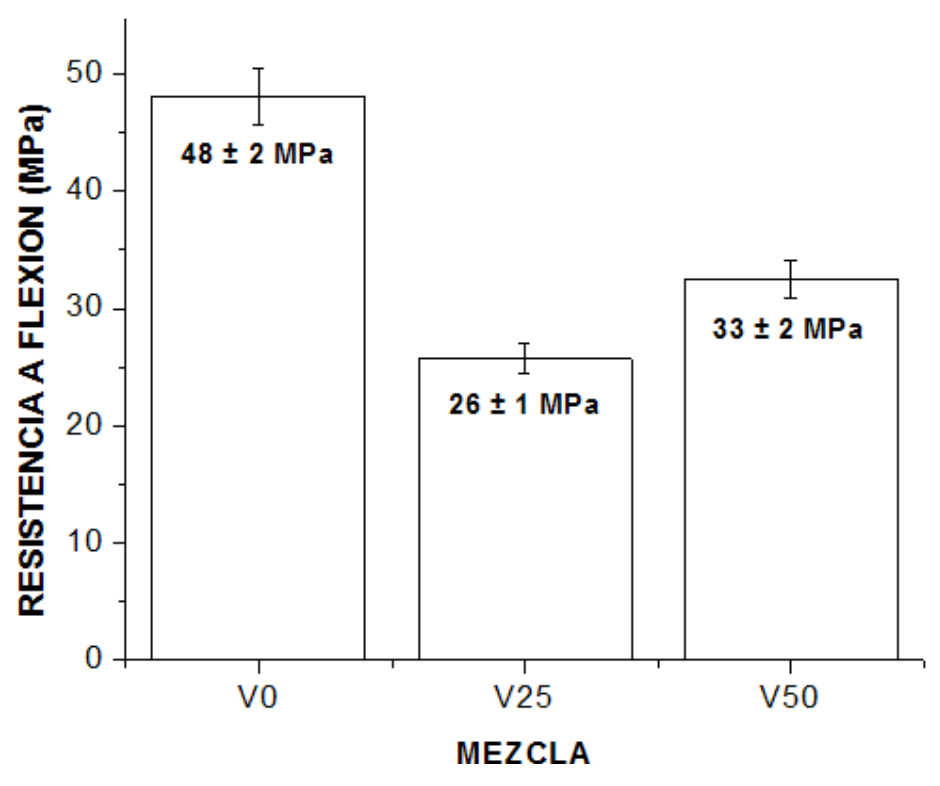

Figura 4. Resistencia a la flexión de las piezas cocidas a la temperatura óptima de cocción.

Fuente: elaboración propia. 

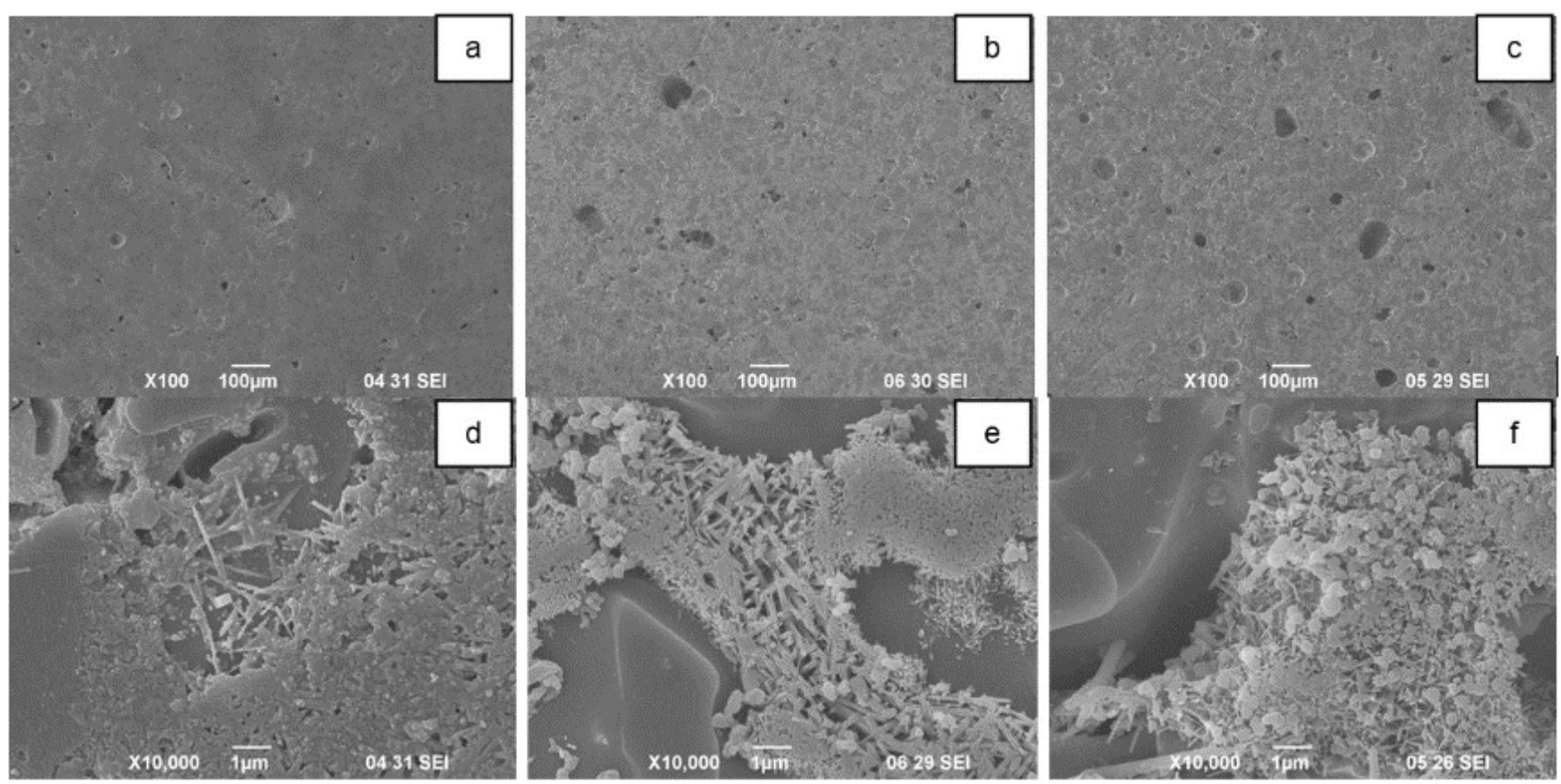

Figura 5. Micrografías MEB en modo SEI de superficies pulidas de probetas cocidas V0 (a), V25 (b) y V50 (c) (100x); y micrografías MEB en modo SEI de superficies pulidas de probetas cocidas V0 (d), V25 (e) y V50 (f) (10000x).

Fuente: elaboración propia.

formados en la superficie externa de los aglomerados de arcilla caolinítica y/o caolín pueden crecer y transformarse en cristales de mullita secundaria, si estos se encuentran cercanos a una matriz de menor viscosidad.

\section{CONCLUSIONES}

La incorporación de polvo de vidrio como remplazo del feldespato en mezclas de gres porcelánico contribuye a la disminución de la temperatura de máxima densificación de los especímenes cocidos en $150{ }^{\circ} \mathrm{C}(\mathrm{V} 25$ y V50), respecto a una composición estándar, comportamiento que se puede atribuir al contenido de óxidos alcalinos $\left(\mathrm{Na}_{2} \mathrm{O}\right)$ y alcalinotérreos $(\mathrm{CaO})$ presentes en el polvo de vidrio, ocasionando la formación de una fase vítrea de menor viscosidad que debería fluir fácilmente llenado los poros; sin embargo, habría una incoherencia con lo anterior ya que la densidad aparente en el material sinterizado disminuye.

Aunque el polvo de vidrio mostró ser un fundente fuerte, los especímenes cocidos en composiciones de gres porcelánico, en donde el feldespato fue sustituido por polvo de vidrio en $25 \%$ y $50 \%$ en peso total (V25 y V50), no permiten obtener gres porcelánico Bla (resistencia a flexión > 35 MPa y absorción de agua $<0,5 \%$ ) y se clasificarían como semi-gres perteneciente al grupo Blla (resistencia a flexión > 22 MPa y absorción de agua 3 - 6\%) y gres perteneciente al grupo Blb (resistencia a flexión > 30 MPa y absorción de agua 0,5-3\%), respectivamente.

\section{FINANCIAMIENTO}

Universidad del Valle (Colombia), y COLCIENCIAS por el apoyo brindado para la financiación y el desarrollo de este estudio, sin lo cual no habría 
podido desarrollarse este proyecto. En particular, a la investigación "Cerámicas Triaxiales basadas en Cenizas del Tamo de Arroz" código 110652128358 apoyada por COLCIENCIAS, Convocatoria 521 de 2010, contrato RC. No. 325-2011.

\section{AGRADECIMIENTOS}

Los autores quieren agradecer a la Universidad del Valle (Colombia), y a COLCIENCIAS por el apoyo brindado para la financiación y el desarrollo de este estudio, sin lo cual no habría podido desarroIlarse este proyecto.

\section{REFERENCIAS}

Andreola, F.; Barbieri, L.; Corradi, A., \& Lancellotti, I. (2007). CRT glass state of the art: A case study: Recycling in ceramic glazes. Journal of the European Ceramic Society, 27(2-3), 1623-1629.

Andreola, F.; Barbieri, L.; Karamanova, E.; Lancellotti, I., \& Pelino, M. (2008). Recycling of CRT panel glass as fluxing agent in the porcelain stoneware tile production. Ceramics International, 34(5), 1289-1295.

Biffi, G, O. (2002). Gres porcelanato: manual de fabricação e técnicas de emprego. São Paulo, Brasil: Faenza Editrice do Brasil Ltda.

Braganca, S., \& Bergmann, C. (2004). Traditional and glass powder porcelain: technical and microstructure analysis. Journal of the European Cermic Society, 24(8), 2383-2388.

Braganca, S.; Bergmann, C., \& Hubner, H. (2006). Effect of quartz particle size on the strength of triaxial porcelain. Journal of the European Ceramic Society, 26(16), 3761-3768.

Carty, W., \& Senapati, U. (1998). Porcelain—Raw materials, processing, phase evolution and mechanical behavior. Journal of the American Ceramic Society, 81(1), 3-20.

Cuéllar Lozano, J. (2000). Desarrollo y caracterización de un gres porcelánico. Tesis de maestría, Universidad Autónoma de Nuevo León.

Dana, K.; Dey, J., \& Das, S. (2005). Synergistic effect of fly ash and blast furnace slag on the mechanical strength of traditional porcelain tiles. Ceramics International, 31(1), 147-152.

Das, S., \& Dana, K. (2003). Differences in densification behaviour of $\mathrm{K}, \mathrm{Na}$ feldspar containing porcelain bodies. Thermochimica Acta, 406(1-2), 199-206.

De Noni Jr., A.; Hotza, D.; Cantavella, V., \& Sánchez, E. (2008). Analysis of the development of microscopic residual stresses on quartz particles in porcelain tile. Journal of the European Ceramic Society, 28(14), 2629-2637.

De Noni Jr., A.; Hotza, D.; Cantavella, V., \& Sánchez, E. (2010). Influence of composition on mechanical behaviour of porcelain tile. Part I: Microstructural characterization and developed phases after firing. Materials Science and Engineering: A, 527(78), 1730-1735.

Guzmán, A.; Delvasto, S.; Sánchez, E., \& Amigó, V. (2013). Cenizas del tamo de arroz como substituto del feldespato en la fabricación de cerámica blanca. Boletín de la Sociedad Española de Cerámica y Vidrio, 52(1), 25-30.

Luz, A., \& Ribeiro, S. (2007). Use of glass waste as a raw material in porcelain stoneware tile mixtures. Ceramics International, 33(5), 761-765.

Márquez, J.; De la Torre, A.; Aranda, M.; Rincón, J., \& Romero, M. (2009). Evolution with temperature of crystalline and amorphous phases in porcelain stoneware. Journal Of the American Ceramic Society, 92(1), 229-234.

Márquez, J.; Rincón, J., \& Romero, M. (2008). Effect of firing temperature on sintering of porcelain stoneware tiles. Ceramics International, 34(8), 1867-1873.

Márquez, J.; Rincón, J., \& Romero, M. (2010a). Effect of microstructure on mechanical properties of porcelain stoneware. Journal of the European Ceramic Society, 30(15), 3063-3069.

Márquez, J.; Rincón, J., \& Romero, M. (2010b). Mullite development on firing in porcelain stoneware bodies. Journal of the European Ceramic Society, 30(7), 1599-1607.

Matteucci, F.; Dondi, M., \& Guarini, G. (2002). Effect of soda-lime glass on sintering and technological properties of porcelain stoneware tiles. Ceramics International, 28(8), 873-880. 
Mattyasovszky-Zsolnay, L. (1957). Mechanical strength of porcelain. Journal of the American Ceramic Society, 40(9), 299-306.

Norma ASTM C326 (2009). Standard Test Method for Drying and Firing Shrinkages of Ceramic Whiteware Clays. American Society for Testing and Materials.

Norma ASTM C329 (1988 (2006)). Standard Test Method for Specific Gravity of Fired Ceramic Whiteware Materials. American Society for Testing and Materials.

Norma ASTM C373 (1988 (2006)). Standard Test Method for Water Absorption, Bulk Density, Apparent Porosity, and Apparent Specific Gravity of Fired Whiteware Products. American Society for Testing and Materials.

Norma ASTM C674 (1988 (2006)). Standard Test Methods for Flexural Properties of Ceramic Whiteware Materials. American Society for Testing and Materials.

Norma ISO 13006 (1998). Ceramic tiles-Definitions, classification, characteristics and marking. International Organization for Standardization.

Raimondo, M.; Zanelli, C.; Guarini, G.; Dondi, M.; Fabbroni, R., \& Cortesi, T. (2009). Process of pyroplastic shaping for special-purpose porcelain stoneware tiles. Ceramics International, 35(5), 1975-1984.

Raimondo, M.; Zanelli, C.; Matteucci, F.; Guarini, G.; Dondi, M., \& Labrincha, J. (2007). Effect of waste glass (TV/PC cathodic tube and screen) on technological properties and sintering behaviour of porcelain stoneware tiles. Ceramics International, 33(4), 615-623.

Rambaldi, E.; Carty, W.; Tucci, A., \& Esposito, L. (2007). Using waste glass as a partial flux substitution and pyroplastic deformation of a porcelain stoneware tile body. Ceramics International, 33(5), 727-733.

Ríos R, C. (2009). Uso de materias primas colombianas para el desarrollo de baldosas cerámicas con alto grado de gresificación. Tesis de maestría, Universidad Nacional de Colombia, Sede Medellín.
Sánchez, E. (2002). Technical considerations on porcelain tile products and their manufacturing process. En M. o. In Qualicer 2002 (ed.), (pp. 57-83). Castellón. Recuperado el 22 de 01 de 2012, de: http://www.qualicer.org/recopilatorio/ponencias/ pdf/0211030s.pdf

Sánchez, E. (2003). Considerações Técnicas sobre Produtos de Revestimento Porcelânico e seus Processos de Manufatura. Parte I. Revista Cerâmica Industrial, 8(2), 7-16. Recuperado el 18 de 01 de 2011, de: http://www.ceramicaindustrial.org.br/main.php

Sánchez, E.; Ibáñez, M.; García-Ten, J.; Quereda, M.; Hutchings, I., \& Xu, Y. (2006). Porcelain tile microstructure: implications for polished tile properties. Journal of the European Ceramic Society, 26(13), 2533-2540.

Stathis, G.; Ekonomakou, A.; Stournaras, C., \& Ftikos, C. (2004). Effect of firing conditions, filler grain size and quartz content on bending strength and physical properties of sanitaryware porcelain. Journal of the European Ceramic Society, 24(8), 2357-2366.

Tucci, A.; Esposito, L.; Rastelli, E.; Palmonari, C., \& Rambaldi, E. (2004). Use of soda-lime scrap-glass as a fluxing agent in a porcelain stoneware tile mix. Journal of the European Ceramic Society, 24(1), 83-92.

Yuruyen, S., \& Toplan, H. (2009). The sintering kinetics of porcelain bodies made from waste glass and fly ash. Ceramics International, 35(6), 2427-2433.

Zanelli, C.; Raimondo, M.; Dondi, M.; Guarini, G., \& Tenorio, P. (2004). Sintering mechanisms of porcelain stoneware tiles. En M. o. Qualicer 2004 (ed.), (pp. 247-259). Castellón. Recuperado el 22 de enero de 2011, de: http://aulavirtual.camaracastellon. com/qualicerCD/pdf/0413191e.pdf

Zanelli, C.; Raimondo, M.; Guarini, G., \& Dondi, M. (2011). The vitreous phase of porcelain stoneware: composition, evolution during sintering and physical properties. Journal of Non-Crystalline Solids, 357(16-17), 3251-3260.

\section{(c) $(1) \Theta$}

